\title{
Temeswar und das Banat in der Temesvarer Zeitung
}

\author{
Eszter JÁNOS \\ Christliche Universität Partium \\ Abteilung für Germanistik \\ szabo.eszter.szidonia@gmail.com
}

\begin{abstract}
Abstrakt. Die Temesvarer Zeitung wirkte seit ihrer Gründung in einer multikulturellen Gesellschaft (Ungarn, Deutsche, Serben, Rumänen, Juden). Diese Heterogenität der Bevölkerung und deren kulturelle Vielfalt spiegeln sich auch in ihrem Kulturteil wider, in dem sich literarische Texte, Essays, Berichte, Kommentare und kritische Besprechungen befinden. Die Forschung konzentriert sich auf die von Adolf Sternberg geleitete Periode (1871-1882), da er dem Blatt einen wichtigen Impuls gab, indem er auf das Feuilleton der Zeitung Akzent legte und das Niveau des Presseorgans mit literarischen und kulturellen Beiträgen heben wollte.
\end{abstract}

Schlüsselwörter: Temesvarer Zeitung, multikulturellen Gesellschaft, Heterogenität der Bevölkerung, Texte, Essays, Berichte, Kommentare, kritische Besprechungen

\begin{abstract}
Since its founding, the Temesvar newspaper (Temesvarer Zeitung) has been part of a multicultural society (Hungarians, Germans, Serbs, Romanians, Jews). This heterogeneity of the population and its cultural diversity is also reflected in its cultural section, which contains literary texts, essays, reports, commentaries and critical discussions. The research focuses on the period headed by Adolf Sternberg (1871-1882), as he gave an important impetus by putting emphasis on the features page of the newspaper and wanted to raise the level of the press organ with literary and cultural contributions.
\end{abstract}

Keywords: Temesvar newspaper (Temesvarer Zeitung), heterogeneity of the population, multicultural society, literary texts, essays, reports, commentaries, critical discussions 


\section{Plaudereien, Genrebilder, Bagatellen in der Temesvarer Zeitung}

Die Redaktion der Zeitung strebte nach der Hochhaltung eines hohen Niveaus im Feuilleton; dazu trugen selbstverständlich die politische Stabilität und auch eine gewisse Kontinuität in der Tätigkeit eines einzigen Redakteurs bei. Adolf Sternberg betonte mehrmals in dieser Periode, dass die Zeitung die Banater Gegend und die Stadt mit speziellem Interesse betrachtet. Mit harmlosem Scherz und frischem Ton wird auf Übelstände, Fahrlässigkeiten und Missbräuche hingewiesen und die Aufmerksamkeit der maßgebenden Personen und Kreise auf Dinge gelenkt, die sich derselben sonst vielleicht entzogen hätten, teilt der Redakteur den Lesern seine Ziele mit. Außer den bisher dargestellten Themen des Feuilletons (Fortsetzungsromane, Novellen, Erzählungen, Reiseskizzen, Völkerbilder) war noch der lokale Teil bedeutend, der in verschiedenen Formen „unter dem Strich“ vorkam: Temesvarer Plaudereien, Temesvarer Genrebilder, Temesvarer Bagatellen, Temesvarer Raritäten, Temesvarer Nächte, Temesvarer Bilder und Geschichten. In den Temesvarer Nächten, die Sternberg auch regelmäßig verfasste, verlockte er die Leser mit der Fortsetzungsstruktur, wie sie im Falle der Fortsetzungsromane schon bekannt war. Nach einer spannenden Situation, die am Ende der Fortsetzung etabliert wird, bleibt die Geschichte offen und wird erst in der nächsten Fortsetzung gelöst.

Den größten Teil dieser Texte bilden die Plaudereien, in denen der Feuilletonist über die wichtigsten Ereignisse der Stadt und der Region berichtet, was die meisten Menschen ohne seine Mitwirkung nicht bemerken würden. Innerhalb des Feuilletons stellt die Plauderei in formaler Hinsicht eine leichtere Gattung als Essay, Aufsatz und Betrachtung dar, worin das Belustigende vorherrscht. Obwohl keine eindeutige und einheitliche Definition über die Plauderei existiert, sind sich die Untersuchungen in mehreren Punkten einig, und zwar, dass sie schwer zu definieren sei. Das kritische Räsonnement und die subjektive Meinung des Journalisten gehörten im Allgemeinen zu den Charakteristiken des Feuilletons, des Artikels „unter dem Strich“. Auch die Unterhaltung durch das Feuilleton zählte zur Betonung des Unterschieds zwischen den politischen Artikeln und den Veröffentlichungen ,unter dem Strich, oft um den Negativismus der anderen Nachrichten auszugleichen (Stöber 2005. 203). Hieronymus Lorm definierte 1848 diesen Unterschied folgendermaßen: Das Feuilleton kann auch persönliche Meinungen beinhalten, während die politischen Artikel die öffentlichen Ansichten, also „die Meinung Aller“ (Tóth 2003. 216) vertreten sollen. Inhaltlich gibt es auch einen krassen Unterschied zwischen dem Teil „über dem Strich“ und „unter dem Strich“; das Feuilleton befasst sich mit den Themen der Kunst, Literatur und Philosophie, und nicht mit den aktuellen politischen Fragen, mit den Tagesereignissen. 
Diese persönliche Art des Sehens und Schilderns der Welt(-ereignisse) und die persönliche Art des Folgerns und Schlüsseziehens ist ein wesentliches Charakteristikum des Feuilletons, und damit auch der Plauderei. Wegen dieser starken Subjektivität der Gattung definiert man sie oft vorurteilshaft, vor allem nach den alten Vorstellungen als „Tändeleien“ (Todorow 1996. 3), als eine leichte, witzige, amüsante, oberflächliche Wortgaukelei. Auf einer Seite ist diese Pauschalierung berechtigt; das Feuilleton kann „belanglos geschwätzig Artikel“ beinhalten; auf der anderen Seite aber kann es auch ernste, relevante ,alltagskulturelle, künstlerische und wissenschaftliche Vermittlungsaufgaben erfüllen und ein bedeutendes Diskursfeld für öffentliches Nachdenken und ästhetische oder gesellschaftspolitische Innovationen ausbilden" (Todorow 1996. 4).

Mehrere solcher Artikel findet man auch in der Temesvarer Zeitung, die als Plaudereien, Genrebilder, Bagatellen, Raritäten, Bilder und Geschichten betitelt wurden. Sogar die Benennungen deuten auf den Status dieser Texte innerhalb der Zeitung hin; es werden hier interessante Begebenheiten der Stadt im kritischen, ironischen Stil aus der Perspektive eines Feuilletonisten dargestellt. Die Plaudereien stammen hauptsächlich vom Chefredakteur der Zeitung Adolf Sternberg, der den Lesern die Dinge so zeigt, wie „sie sich in seiner Persönlichkeit widerspiegeln“; er beleuchtet alles „mit den Strahlen seiner individuellen Stimmung“, und „er verräth überall die Theilnahme an dem Gegenstande“ (Eckstein 1876, zit. nach Jäger 1988. 54). In diesem Merkmal, „im Durchschimmern der Subjectivität" sieht Eckstein das Wesen des Feuilletons und der dazugehörigen Textsorten (Plaudereien, Skizze, Humoreske usw.), und dieses herrscht auch über die lokalen Artikel der untersuchten Zeitung vor. Diese Art der Darstellung eines Themas, also die Wochenplauderei nennt Benedek Tóth ein kontinuierliches fiktives Gespräch in der Zeitung, oder den Dialog ,unter dem Strich“. Er untersucht diese Gattung als spezifischer Diskurs, wobei die wichtigsten Kriterien der Analyse die Unterschiede zwischen Subjektivität und Objektivität und zwischen der Realität und der Fiktion sind. Auf den subjektiven Standpunkt des Feuilletonisten, der immer die Hauptrolle seiner Artikel spielt, deuten auch die oben erwähnten Autoren (Haacke, Todorow, Eckstein) hin. Benedek Tóth bezeichnet als ein einziges äußeres „objektives“ Merkmal der Wochenplauderei, dass sie „unter dem Strich“ sei.

Die spezifische Textsorte des Feuilletons ist auch deswegen problematisch, weil sie die Grenze zwischen der Realität und der Fiktion ständig überschreitet. Die Situation und der Status des Feuilletons sind eindeutig, wenn darin literarische Texte (Gedichte, Novellen, Fortsetzungsromane, aber auch Reisebeschreibungen) erscheinen. Obwohl sie in der Zeitung veröffentlicht werden, gehört das nicht zu ihrer Charakteristik; ihre Erscheinung in der Zeitung ist kein inhärenter Aspekt (Tóth 2014. 463). Im Gegensatz dazu kommt die Wochenplauderei in der Zeitung zustande, was auch ihr spezifisches Merkmal ist. 
Das inhaltliche Spektrum dieser Plaudereien in der Temesvarer Zeitung entspricht ungefähr der Wiener Wochenplauderei, deren Anfänge in den biedermeierlichen Unterhaltungszeitungen liegen. In diesen Rubriken wurden „,in locker zusammenhängender Form“ die Ereignisse in Wien dargestellt. Die Wiener Theaterzeitung erwähnte Themen, mit denen sich 1820 die Briefe über Wien beschäftigten:

Am willkommensten sind Anekdoten des Tages, Begebenheiten, Vorfälle, lustige und tragische Ereignisse, Beschreibungen von Volks- und Bürgerfesten, Moden, Trachten, Anzeigen von der Ankunft und Abreise berühmter Personen, Sterbefälle, Leichenbegängnisse, etc. kurz Gegenstände, welche die gebildete Welt unmittelbar angehen und welche zur Belehrung, Aufheiterung und Bekanntmachung geeignet sind (Kauffmann 2000. 345).

Kauffmann behauptet, dass diese Rubriken erst dann ihren Höhepunkt erreicht hatten, als sie sich unter dem Einfluss der französischen Chronique einen eigenen Stil, und zwar den ironischen, witzigen Stil der Causerie, der Plauderei herausbildeten. Mitte der vierziger Jahre spricht man in den Wiener Unterhaltungszeitungen über Wochenplaudereien im moderneren Sinn. Sie bleibt auch nach der Revolutionszeit der Ort, wo das Individuum subjektiv, wenn auch ironisch, kritisch seine Meinung formulieren kann.

\section{„Temesvar ist (k)eine gewöhnliche Provinzstadt“"1}

In der untersuchten Periode erschienen in der Temesvarer Zeitung mehrere Plaudereien und ähnliche Textsorten (Genrebilder, Bagatellen, Raritäten, Nächte usw.), in deren Zentrum hauptsächlich die Geschehnisse der Stadt und des Theaters in Temeswar stehen. Sie wurden nicht regelmäßig wöchentlich veröffentlicht, sondern die Zeitung brachte monatlich einen Artikel zu dieser Thematik. Es werden in diesen Texten vor allem spannende Geschichten erzählt; die Darstellung von komplexen Charakteren oder die Veranschaulichung ihrer inneren Entwicklung ist weglassbar. Die große Themenvielfalt des lokalen Teils der Zeitung verhindert die einheitliche Behandlung des Stoffes, was aber auch charakteristisches Merkmal der Gattung ist. Die Themen variieren von den Ehe- und Liebesgeschichten über die Astrologie bis zum Theater, zu den verschiedenen Reiseskizzen oder Anekdoten. In der Plauderei berührt der Feuilletonist ein Thema nur oberflächlich und meistens ironisch; er darf sich mit einem Stoff nicht

1 Sternberg, Adolf: An das Temesvarer Lesepublikum. Temesvarer Zeitung Nr. 186 vom 15. August 1886. S. 1. 
ernst und ausführlich beschäftigen. Also spielt die Digression, die bewusste Abschweifung von einem Thema zum anderen eine wichtige Rolle in dieser Gattung (Kauffmann 2000. 351). Trotz des digressiven Status dieser Textsorte zeichnet sich in diesen Artikeln ein Thema aus, womit der Feuilletonist sich mehrmals beschäftigte, und zwar mit dem Status der Stadt, ob sie die charakteristischen Züge einer Kleinstadt oder Großstadt trägt.

Im Mai 1871 stellte sich Adolf Sternberg als neuer Redakteur der Temesvarer Zeitung vor und formulierte auch seine Offenheit gegenüber der Stadt:

Man hat mir so viel Schönes und Rühmenswerthes von der Intelligenz und den kritischen Anforderungen des hiesigen Publikums erzählt, und die Ansprüche desselben so hoch veranschlagt, daß diese Unruhe recht begreiflich ist, bei allem Selbstgefühl, an welchem es und Journalisten sonst gerade nicht zu mangeln pflegt (Temesvarer Zeitung 1871/115. 2)

Die lobenden Worte über die Stadt wurden mit Hilfe einer Anekdote bestätigt, die der neue Redakteur dem Publikum vorstellt: es geht um ein Gespräch in Pest auf dem Elisabethplatz, wo sich Sternberg mit einer Temeswarerin über seine neue Arbeit unterhält. Nach einem Missverständnis, wobei die Frau ihren Gesprächspartner falsch verstanden hat, dass er nicht nach Temeswar, in die Provinz fahren will, rühmte sie Temeswar wegen ihrer kulturellen Vielfältigkeit:

Ich kann Ihnen die Versicherung geben, daß unsere Gesellschaft die Kreise weit aufwiegt, mit denen Sie zu verkehren pflegen, und noch Eines muß ich Ihnen sagen, [...] Sie müssen sich sehr anstrengen, und das in jeder Beziehung, in literarischer sowohl, als auch in gesellschaftlicher Beziehung, um bei uns zu reussiren (Temesvarer Zeitung 1871/115. 2).

Es wäre auch nicht verblüffend gewesen, wenn sich Sternberg für die weite Stadt der Monarchie nicht hätte engagieren wollen. Eine ähnliche Geschichte ist die des Kulturkritikers, Arztes und Zionisten Max Nordau, ${ }^{2}$ der Mitarbeiter des Pester Lloyd war. Nordau ${ }^{3}$ hatte sich für das außergewöhnlich hohe Gehalt von 400 Gulden pro Monat vom Herausgeber des Pester Lloyd als Korrespondent

2 Max Nordau (1849-1923), Arzt, Zionist und kulturkritischer Essayist war zwischen 1867 und 1876 neben seinem Medizinstudium für den Pester Lloyd als Feuilletonist tätig. In diesem Amt verweilte er von April bis November 1873 in Wien und belieferte das Organ mit ca. 100 Depeschen, Feuilletons und Berichten. Er publizierte insgesamt cca. 230 Veröffentlichungen, wovon die knappe Hälfte in die Wiener Zeit fiel. Nach Ujvári, Hedvig: Feuilletons über die Wiener Weltausstellung 1873 im Pester Lloyd. http://www.kakanien-revisited.at/beitr/fallstudie/ Hujvari1.pdf (Zugriff am 17. 07. 2016)

3 Zur Nordau-Forschung siehe ausführlich die Publikationen von Hedvig Ujvári: Dekadenzkritik aus der „Provinzstadt“: Max Nordaus Pester Publizistik. Budapest 2007. 
für die Weltausstellung engagieren lassen, die am 1. Mai 1873 in Wien eröffnet wurde. Nordaus Feuilletons erregten das Interesse des Lesepublikums, aber Nordau, karrierebewusst und wienzentriert, schrieb seiner Schwester voller Enttäuschung, bei Hofe lese man die „alte“ und die Neue Freie Presse, die Wiener Hauptstadtblätter, nicht aber den Pester Lloyd, der sei, so Nordau, ein „Provinzblatt“" (Ehrenpreis 2006. 1800).

Ob Sternberg Temeswar für eine Provinzstadt hält oder nicht, stellt sich aus seinen Artikeln nicht eindeutig heraus, obwohl er mehrmals kritisch auf die Mangelhaftigkeit der Stadt hinweist. In diesen Artikeln stellt Sternberg in humoristisch-satirischem Stil dem Publikum seine Wahrnehmungen über die Stadt vor. An dem Tag seiner Ankunft beschwert er sich über die missliche Situation im Hotel: „Ich schlief in dem Gedanken ein, daß Temesvar Hotels besitze, die kaum die primitivsten Anforderungen erfüllen, welche man an derartige Etablissements zu stellen berechtigt ist", und darüber, dass das Stubenmädchen kein Französisch sprach: „Ich gab keine Antwort. [...] Das Stubenmädchen hat seit diesem Momente eine solche Scheu vor mir, wie ich vor den Temesvarer Hotels, und das will viel sagen“ (Temesvarer Zeitung 1871/116. 1). Über eine ähnliche Stimmung berichtet er auch in dem nächsten Artikel: „Die Wellen der Bega rauschten unheimlich, der Himmel war grau und düster und ein feiner, kalter Regen begann zu fallen“ (Temesvarer Zeitung 1871/119. 2).

Sternberg wollte das Niveau der Temesvarer Zeitung heben; er schrieb Gedichte, Essays, Tagesnotizen und Leitartikel. Zu feierlichen Anlässen, seien sie nun religiös oder politisch, verfasste er vier- bis fünfmal Leitartikel. Ein solcher Artikel ist auch der nächste Beitrag von ihm; betitelt wurde er mit Temesvarer Plaudereien und ist an Pfingsten 1871 erschienen. Die Titelwahl weist auf den Charakter des Artikels hin, es geht um ein Straßengespräch, aus dem man wichtige Informationen über den Geschmack der Leser erhält.

Wie gefällt Ihnen die Temesvarer Zeitung jetzt?, fragte der Eine.

Nicht schlecht, gab der Andere zur Antwort, nur hätte ich gewünscht, daß sich das Blatt mehr mit den lokalen Interessen beschäftige.

Was fällt Ihnen ein, gab der Zweite zur Antwort, ich mache ihr gerade den Vorwurf, daß sie sich zu viel um solche Lapalien kümmert, die schließlich nur einen sehr geringen Kreis interessieren. Politik ist heute die Losung des Tages und zwar die höhere, und um Anderes kümmere ich mich keinen Pfifferling.

Haben Sie den neuen Redakteur schon gesehen?

Nein, aber man hat mir gesagt... 
Was kann man Ihnen gesagt haben? warf der Zweite ein, es gibt nur Eine Zeitung, die diesen Namen verdient und nur Einen Redakteur, von dem es überhaupt der Mühe werth ist, zu reden. Ich habe die Neue freie Presse im Sinne, ich sage Ihnen, ich habe in der Beilage dieses Blattes für Völkerkunde gestern einen Artikel gelesen, einen Artikel, sage ich Ihnen, der schon das Höchste ist, was es gibt. Er heißt: Die Taucherglocke, und da wird erzählt, daß es Menschen gibt, die sich in eine metallene Glocke hineinsetzen, die unten ganz offen ist, und sich so auf den Meeresgrund hinablassen, ohne zu ertrinken.

Sonst wäre es auch eine Dummheit sich hinabzulassen, entgegnete der Zweite.

Ja, aber sie können nicht ertrinken, wegen der komponirten Luft, die das Wasser nicht in die Glocke läßt.

Komprimirte Luft, wollen Sie sagen, warf der Zweite mit einem stolzen Lächeln ein.

So! nun ich werde in der Neuen freien Presse nachsehen, wie es geschrieben ist, denn für mich ist die Neue freie Presse Autorität und nur ihr Redakteur ist ein Redakteur, sonst sind alle Redakteure... Ich fühlte einen mächtigen Reiz, laut aufzulachen, da aber fiel es mir ein, daß der Verehrer der Neuen freien Presse vielleicht auch Recht haben könne, und in Rücksicht auf das Wetter der Pfingstfeiertage bezähmte ich meinen Kitzel (Temesvarer Zeitung 1871/122. 2).

In seinen Genrebildern vergisst er auch nicht den literarischen, theatralischen oder musikalischen Geschmack der Menschen in Temeswar zu kritisieren:

So viel steht fest, wenn mich Etwas von Temesvar zu vertreiben fähig wäre, so ist es nicht das schlechte Wasser, nicht die, wie man sagt, ungesunde Luft, nicht Albert Strasser oder einer der sonstigen Vorzüge dieser Stadt, sondern lediglich die musikalische Pest, die hier in bedenklichster Weise grassirt und gegen die man bisher weder Desinfektions- noch sonstige Schutzmittel zur Anwendung gebracht hat. Man hat prophylaktische Mittel gegen die Cholera, die Blattern, ja sogar gegen die Viehseuche; warum überliefert man also eine unschuldige Generation gerade diesem entsetzlichen Übel? Man sagt, daß Temesvar verhältnismäßig eine größere Anzahl geistesgestörter Personen habe, als andere Städte und zerbricht sich den Kopf über die Ursache dieser betrübenden Erscheinung. Ich kenne dieselbe und empfehle meine Entdeckung den Psychiatriden: die Unglücklichen sind durch die Musik verrückt geworden (Temesvarer Zeitung 1871/147. 2). 
Außer den musikalischen Leiden ärgerte sich der Feuilletonist über das Angebot des Theaters, das er mit harter Kritik beschrieb, indem er zur Verbesserung des Repertoires und zum Gedeihen der Unternehmung eines neuen Theaterbaues ermutigte. In Temeswar wurden nach Sternberg die primitivsten Anforderungen des Geschmacks und Piecen von einer geradezu ekelhaften Frivolität gespielt, wobei überall sittliche Unzulässigkeit, Geschmacklosigkeit und Blödsinn herrscht. Die Rolle wahrhaft künstlerischer Bestrebungen nahmen gymnastische Produktionen ein und auf dem vernachlässigten Boden der darstellenden Kunst machte sich das Unkraut des Volkssängertums in einer Weise breit, welche zu den ernstesten Bedenken Anlass gibt. Solche Produktionen beeinflussen das Publikum; sie bringen es in eine falsche Richtung, sie haben eine verwildernde, verdummende Wirkung auf die Zuschauer, sie „stumpfen den Rest ästhetischen Gefühles ab, dessen sich der Mensch nie ganz entschlagen soll und verbreiten eine Rohheit des Geschmacks und der Sitten, welche geradezu als verderblich betrachtet werden muß" (Temesvarer Zeitung 1871/167. 1). Das beste und einzige "Correktiv“ gegen dieses künstlerische Unkraut ist ein gutes Theater, und Temeswar sollte sein Theater gewissermaßen als ein Palladium betrachten, ,warum wir dem Gedeihen der Unternehmung eines neuen Theaterbaues aus vollem Herzen Glück wünschen müssen“ (Temesvarer Zeitung 1871/167. 1), betont der neue Redakteur. Er vergaß nie über die Schwächen der Stadt ironisch zu berichten; die Straßen seien schlecht, die Gaslaternen schwach und aus diesem Grund sei es in der Stadt finster. Neben diesem ironischen Stil konzentriert sich der Feuilletonist auch darauf, dass der Leser angesprochen wird. Diese Anrede ist auch ein wichtiges Element in der Struktur; sie leiten die neuen Mikronarrativen ein: „Wenn Sie gerade absolut nichts Besseres zu thun haben, setzen Sie sich ruhig in Ihre Sophaecke und lassen sich ein wenig amusieren; ich gebe Ihnen das Versprechen, so wenig langweilig zu sein, als dieses einem Feuilletonisten von ein wenig Styl und Scharfsinn nur möglich ist“ (Temesvarer Zeitung 1872/199. 1). Der Feuilletonist erstellt durch seine ständige Anwesenheit den Zusammenhang zwischen den Plaudereien, den unterschiedlichen und ausschweifenden Geschichten, also den sog. Mikronarrativen, und so entsteht aus diesen Serien eine Makronarrative (Tóth 2003. 220). Das verbindende Element ist in diesem Falle Temeswar, das der neue Redakteur für sich selbst langsam entdeckt, und die Leser betrachten diese Erfahrungen aus seiner Perspektive. Dieses selbstreflexive Verhalten des Feuilletonisten gehört auch zu den Grundmerkmalen einer Plauderei. Seine regelmäßigen Spaziergänge in der Stadt ermöglichten ihm eine neue positive Sichtweise, die er auch zugestand:

Ich hatte mich nämlich bisher immer der Ansicht hingegeben, daß in Temesvar die Welt allenthalben mit Brettern verschlagen ist und erkannte erst jetzt zu meiner Beschämung, daß dieselbe wirklich offen sei und 
daß man faktisch vom Ende der Vorstadt Fabrik bis zu dem der Josefstadt gehen können, ohne irgendwo mit der Nase an das Ende der Welt zu stoßen, von wo man die Füße in das leere Nichts hinabhängen lassen kann (Temesvarer Zeitung 1873/154. 1).

Die Bega mit ihren romantischen Ufern, ihren prachtvollen Weidenbäumen und der ganzen pittoresken Szenerie wurde positiv beschrieben: „Ich war, wie das an schönen Sommerabenden üblich, einigermaßen elegisch gestimmt und deklamirte leise Göthe’s Abendlied“ (Temesvarer Zeitung 1873/154. 1). Durch diese „verborgenbleibende Eingeweihtseins-Vorstellung“ der Peripherie gehört die Kleinstadt, im Gegensatz zu den großstädtisch-hauptstädtischen, zu der homogenisierenden Welt der Metropole, zu den charakteristischen Gebilden Mitteleuropas, behauptet István Fried. Die Kleinstädte besitzen teils hinsichtlich der Lebensqualität, teils hinsichtlich der sich in der Zeit ereignenden Geschehnisse, zahlreiche ähnliche Merkmale. Sie sind beispielweise die fast identische Stadtstruktur mit den einander ähnlichen Theatergebäuden, die aneinander erinnernden Formen der Bahnstationen und der Bahnhofrestaurants und die Platzmusik der Garnisonsstädte am Sonntagvormittag (Fried 2001. 125).

Die Kleinstadt ist Zeuge vieler Ereignisse, die sich dann in den Plaudereien zu einem epischen Geflecht entwickeln. Die Eröffnung des neuen Theaters gab dem Feuilletonisten Anlass, seine Ansichten mit den Lesern zu teilen, und die Kritik darüber verschwand nicht aus den Plaudereien der Temesvarer Zeitung. Sternberg beschuldigte die Direktion wegen des schwachen Besuchs im Theater: „Wenn man sich Tragödinen die nichts taugen von Dem und Jenem aufoktroyiren läßt, wenn man bei den Besetzungen von Rollen, alles Andere eher als die Individualität der Darsteller zu Rathe zieht, so ist der Mißerfolg unausbleiblich“ (Temesvarer Zeitung 1875/273. 1). Nur eine rasche und gründliche Umkehr kann dem Theater noch helfen, „den Mängeln des Personals muß abgeholfen, die Rollen müssen nach einem raisonablen System vertheilt und statt leerer Reklame müssen positive Leistungen geboten werden“ (Temesvarer Zeitung 1875/273. 1), behauptet Sternberg. Die weiteren Defizite der Stadt wurden in den Temesvarer Schattenbildern verfasst. Die vier Teile der Stadt (Festung, Fabrik, Josefstadt und die beiden Meierhöfe) sollten einander näher gerückt sein werden, weil sich so kein ordentliches Ganzes bildet. Weitere Mängel wurden ans Licht gebracht:

Die nationalen Gegensätze und der abnehmende Wohlstand der Bevölkerung theilen diese auch noch in so viele Klassen, daß an ein Aufblühen des gesellschaftlichen Lebens noch lange nicht zu denken ist. Hiezu kömmt noch die großartige Klatschsucht und der liebe Hochmuth, Eigenschaften, die in jeder Provinzstadt zu finden sind, die mir aber nirgends so scharf ausgeprägt vorkamen, als hier (Temesvarer Zeitung 1878/ 23. 1). 
Einige negative Aspekte wiederholten sich, wie der Zustand der Straßen oder die Schwäche der Beleuchtung; die Gebäude im Zentrum wurden aber wegen ihrer Schönheit gepriesen. Im Innern der Häuser sieht es zuweilen auch traurig aus; man trifft oft hölzerne Stiegen, schmutzige Gänge, überhaupt Unreinlichkeit an. Die Gespräche und Handlungen der Leute unter sich beobachtend herrscht eine unglaubliche Ungeniertheit, die oftmals an Zynismus streift. Auch der groBe Anteil der Bettler verdirbt das Bild der Stadt und rückt sie dem Status einer Provinzstadt näher.

Der Domplatz hat auch keine Anziehungskraft; kahl und nackt bietet er sich dem Auge des Beschauers dar, wenn nicht gerade Markt ist. Die Statuen dürften nicht so vernachlässigt werden; sie würden mehr Aufmerksamkeit verdienen, steht in der Plauderei. Altmodisch scheinen die Omnibusse, „diese Vehikel, ein Überbleibsel besserer Zeiten passen aber in die Jetztzeit ebensowenig, wie Folterinstrumente, mit denen sie Ähnlichkeiten besitzen sollen“ (Temesvarer Zeitung 1878/23. 1). Die Kälte im Theater (auf der Bühne und im Zuschauerraum) wurde auch erwähnt, „ein Umstand, der ganz geeignet ist, einem den Theaterbesuch gründlich zu verleiden“.

Der „Skribler“ der Plauderei berichtete über einen einigen Platz positiv in Temeswar: über den Stadtpark, den die Einheimischen nicht genug schätzen: „Ich kenne die Umgebungen von Wien und Graz genau, die reizende Margarethen-Insel bei Budapest, die Moldau-Inseln bei Prag, den Brünner Augarten, ich muß aber aufrichtig bekennen, daß der hiesige Stadtpark sehr hübsche Partien hat" (Temesvarer Zeitung 1878/23. 1). Über den Park wurde auch früher von Sternberg geschrieben; er sah ihn als die einzige Oase in der Stadt: „Temesvar wäre faktisch zu einem bleibenden Aufenthalt ungeeignet, wenn außerhalb seiner dumpfen Mauern und schlammigen Gräben, mitten in einer Staubwüste, nicht dieser reizende kleine Fleck wäre, wo Natur und Kunst ihre schönsten Reize entfalten“ (Temesvarer Zeitung 1872/148. 1).

Ähnliche kleinstädtische Reaktionen beschrieb auch Adolf (Ötvös) Silberstein im Neuen Freien Lloyd unter dem Titel Federzeichnungen aus der Provinz, worauf Sternberg in einem Artikel der Temesvarer Zeitung reagierte. Bei der Beantwortung der Vorwürfe von Silberstein versuchte der neue Redakteur eine Ehrenrettung der Provinz zu verfassen, in der sich seine Sympathie zur Provinz eindeutig auszeichnete. Silberstein sprach über arrogante, ehrgeizige Provinzler und die Damen wurden von ihm in einer keineswegs galanten Weise vorgestellt: „Auf der Straße erwidern sie kaum den Gruß, der ihnen mit tiefabgezogenem Hute entgegengebracht wird. Aber für treue Liebe ist die Provinzfrau umso empfänglicher" (Temesvarer Zeitung 1871/185. 1). Über die Presseverhältnisse der Provinz hatte er noch eine schlechtere Meinung: „Die Stadt darf sich ohne Zuthun des Redakteurs nicht amusiren. Er muß Bälle arrangiren, bei Konzerten aushelfen, den ersten feierlichen und den letzten humoristischen Toast ausbringen; er tanzt 
wie ein Gott, konversirt wie ein Franzose, singt wie ein Wachtel und trinkt wie ein Schwamm“. Um diese Lästerungen zu mildern suchte Sternberg für jeden Vorwurf einen positiven Satz über die Provinzler, und zwar dass er das Glück gehabt habe, in der Provinz so anständige, bescheidene und wirklich anspruchslose Menschen kennen zu lernen, wie er sie nur in den großen Städten gefunden habe. Die scharfen Kritiken behandeln allgemeinmenschliche Eigenschaften, die sowohl in der Provinz, als auch in der Welt passieren könnten, meint Sternberg. Was die provinzialen Presseverhältnisse betrifft, verzichtete er auf diese Ehre, die Silberstein aufzählt; als eventuelles Beispiel nannte er seinen Konkurrenten Albert Strasser. Die Temesvarer Damen verteidigte der neue Redakteur mit der Begründung: „Ich bin der festen Ansicht, daß der Verfasser diese Lästerungen nicht niedergeschrieben hätte, so lange er sich unter den schönen Augen der hiesigen Damen befand, ein neuer Beweis der Macht derselben, wenn es eines solchen noch bedurft hätte“ (Temesvarer Zeitung 1871/185. 1). In der Ablehnung dieser „frevelhaften Blasphemien“ war Sternbergs Standpunkt eindeutig wahrnehmbar; Temeswar sei keine gewöhnliche Provinzstadt, wie er später die Stadt nannte. Als Sternberg 1886 nach Temeswar zurückkehrte, begrüßte er sein altes Publikum mit den folgenden Worten:

Temesvar ist keine gewöhnliche Provinzstadt; dasselbe ist noch heute ein Emporium der Intelligenz für einen großen Landesteil und es besitzt eine verhältnismäßig große Anzahl ausgezeichneter Männer, die sich der größten Hochachtung des Landes erfreuen. Um den Beifall solcher Männer und um denjenigen eines so intelligenten Publikums zu ringen, wie es das Temesvarer ist, ist wohl ein Ziel der Mühen und kräftigen Strebens wert (Temesvarer Zeitung 1886/186. 1).

\section{„Temesvar ist eine kleine Großstadt“4}

Am Anfang der untersuchten Periode der Temesvarer Zeitung wurden auch solche Feuilletons (Plaudereien, Schattenbilder, Bilder und Geschichten) veröffentlicht, in denen Temeswar als Großstadt bezeichnet wurde. Die Stadt bestand damals aus der inneren Stadt oder Festung und aus den Vorstädten, und hatte eine Bevölkerungszahl von 32.000 und einigen hundert Seelen. Obwohl die kritischen Stimmen über den Mangel der Stadt nicht fehlten, tauchte immer öfter der Wille auf, aus dem Status der Provinzstadt herauszukommen, um eine Großstadt zu werden. Das rege Leben und Treiben sowohl auf materiellem, als auch auf

4 Ein geistreicher Feuilletonist eines hauptstädtischen Blattes machte diese Aussage, als er sich in Temeswar befand. In: Temesvarer Bilder und Geschichten. Temesvarer Zeitung Nr. 179 vom 6. August 1882. S. 1. 
geistigem Gebiete in der Stadt sowie der Ausbau der Eisenbahnlinie Temeswar Orsova legitimierte diesen Wunsch (Temesvarer Zeitung 1872/15. 2). Neben den Fehlentwicklungen und Schwächen der Stadt, die die Einheimischen leichter wahrnehmen, existieren in der Hauptstadt des Banats auch solche Annehmlichkeiten, wie in den anderen Großstädten.

In der täglichen Gewohnheit unserer Umgebung, sind wir zu sehr geneigt, anzunehmen, es müsse Alles so und nicht anders sein, wie es eben ist und der klare Blick des außer Schutzweite stehenden unbefangenen Auges ist uns so fremd, daß unter tausend Temesvarer Einwohnern nicht leicht zehn zu finden sein dürften, die es sich nehmen ließen, Temesvar sei die zweite Stadt des Landes [Hervorhebung von mir - J. E.] (Temesvarer Zeitung 1872/247. 1).

Den Gästen schien die Platzmusik in der Stadt großstädtisch zu sein, und sie stimmten alle darin überein, dass dies keine Stadt in Ungarn mehr zu bieten vermag. In den nächsten Jahren waren die Meinungen der Einheimischen und der Fremden über die Stadt gemischt: Man lobte Temeswar wegen der Unzahl der Brücken; es wurde „kleinwienerisches Elysium“ genannt, und die Kritik des Theaters konnte auch nicht fehlen:

Als ich noch im Temesvarer Theater so manche Vorstellung verschlafte, Pardon verbrachte, so war dies keine Wonne. Auch bei uns in Budapest geht es nicht besser, die deutsche Kunst wandert im Harlekin- und Pollichinellgewande der leichtgeschürzten französischen Operette und das neueste Offenbach'sche Ausstattungsstück macht allabendlich ein leeres Haus (Temesvarer Zeitung 1877/252. 1).

Im Vergleich zu der ungarischen Hauptstadt scheint der großstädtische Status von Temeswar verschwunden zu sein, wobei Budapest immer das nachgeeiferte Vorbild für die Banater Hauptstadt blieb. Die heitere Geselligkeit sogar die französische Leichtlebigkeit in Budapest wirkte anziehend auf die Bewohner im östlichen Teil der Monarchie:

Budapest hat seit den letzten zwei Jahren überraschende Fortschritte gemacht und ist eine wahrhaft königliche Stadt geworden. [...] Wir können weder ein solches Pflaster, noch eine solche Beleuchtung, noch auch solche Prachtbauten haben, wie die Hauptstadt, aber ein Beispiel können wir uns an deren rührigem Streben auf allen Gebieten nehmen und eifrig bemüht sein (Temesvarer Zeitung 1875/180. 1). 
Die öffentlichen Lokale und Spazierplätze sind in Budapest immer überfüllt, während in Temeswar der herrliche Stadtpark, der keiner hauptstädtischen Anlage an Schönheit und geschmackvolleren Anlage nachsteht, „wenn nicht eine Temperatur herrscht, um Eier in der Luft zu backen“ (Temesvarer Zeitung 1875/180. 1), verödet steht. Nolens volens nimmt man den krassen Unterschied zwischen den „Großstädten“ auch dadurch wahr, indem Budapest als „Oben“ bezeichnet wurde.

Die Leute dort Oben haben ein frischeres, lebensmuthigeres Naturell, als wir hier im Banate und daher ihr Leben angenehm genießen, während wir hier faktisch vegetiren und die Langweile des Daseins thatlos über uns ergehen lassen (Temesvarer Zeitung 1875/180. 1).

Es besteht kein Zweifel, dass diese Aussagen über Temeswar nur Halbwahrheiten beinhalten und sie hängen stark davon ab, aus welcher Perspektive sie in Betracht gezogen werden. Budapest gehörte am Ende des 19. Jahrhunderts und in den Jahrzehnten um 1900 zu den am raschesten wachsenden europäischen Großstädten. Diese Tatsache wurde auch in der Plauderei über den Vergleich von Budapest mit Temeswar bestätigt, indem die ungarische Hauptstadt gelobt wurde:

Wo sich früher unscheinbare ein- und zweitstöckige Häuser erhoben, da erstrecken sich jetzt die herrlichsten Paläste in unabsehbarer Reihe, die Radialstraße durchschneidet das Herz der Stadt mit einem breiten Bande von Prachtbauten, der Corso auf dem Donauquai bietet mit seiner prachtvollen Perspektive des Ofner Gebirges, seinem Asphaltpflaster, das an Glätte den Parketten des elegantesten Salons gleichkommt, seiner feenhaften Beleuchtung und der Menge schöner und eleganter Damen, einen Anblick, wie ihn, dem einstimmigen Urtheile erfahrener Reisender zufolge, keine zweite Stadt Europa's [Hervorhebung von mir - J. E.] aufzuweisen hat (Temesvarer Zeitung 1875/180. 1).

Budapest hatte um 1875 ca. 300.000 Bewohner und innerhalb von zwanzig Jahren, d. h. von 1890 bis 1910, stieg die Zahl der Bewohner fast auf das Doppelte, von einer halben auf fast eine Million. Diese rasche Entwicklung der Stadt wurde von Vilmos Heiszler „amerikanisches Tempo“ genannt (Csáky 2010. 277).

Am Ende der 1870er-Jahre vermehrten sich die Ereignisse, die Anlass gaben, stolz auf die Geschichte der Region und Stadt zurückzublicken und sie zu vergrößern. Es geht um zwei bedeutende Angelegenheiten: Die „Centennariumfeier der Reinkorporirung des Banates“ und das Jubiläum der Stadt Temeswar. Das von den Türken zurückeroberte Banat wurde vor hundert Jahren an Ungarn an- 
geschlossen und seit seiner „Reinkorporirung“ wurde die Region wegen seines Reichtums „die Kornkammer Ungarns“:

Das Banat wird wieder das werden, was es war, der reiche Boden einer arbeitsfrohen, intelligenten und patriotischen Bevölkerung, welcher die auf ihn verwendete Mühe hundert- und tausendfältig belohnt und auf welchem jeder arbeitswillige Mensch sein reichliches Auskommen finden wird (Temesvarer Zeitung 1879/93. 1).

In den nächsten ausgewählten Artikeln häufen sich die positiven Selbstbeschreibungen, in denen das Banat und dessen Hauptstadt Temeswar als „Spiegelbild des Makrokosmos des multilingualen und plurikulturellen Königreichs“ (Csáky 2010. 285) fungieren. „Temesvar sei eine kleine Großstadt“ und „in der That dürfte es vielleicht kaum eine zweite Provinzstadt in Ungarn geben, in der sich das eigentlich städtische Element so konzentrirt zeigt" (Temesvarer Zeitung 1882/179. 1), wo sich der Stempel des Provinzmäßigen fast gar nicht erkennen lässt, behauptet ein Feuilletonist eines hauptstädtischen Blattes.

Während der fast hundertjährigen Existenz dieser Stadt ist es den Vorfahren gelungen, „Temesvar auf jene Stufe zu bringen, daß es stolz sein kann, zu den ersten Städten unseres Vaterlandes [Hervorhebung von mir - J. E.] gezählt zu werden“ (Temesvarer Zeitung 1882/190. 2). In der Stimmung dieser Festlichkeit wurden noch weitere Artikel veröffentlicht, die sich mit dem Selbstbild der Stadt beschäftigen:

Temesvar kann mit Recht stolz auf seine Vergangenheit sein. Von wilden Kämpfen aller Art umtost, von Waffenlärm und dem Ansturm der Nationalitäten, von einer Bevölkerung der heterogensten Art in Sprache, Sitten und Aspirationen: Zivilisation, Fortschritt, Patriotismus und sich allezeit als unerschütterlicher und unermüdlicher Kulturträger bewahrt, als vorgeschobenster und fester Punkt europäischer Bildung in dem brausenden Meere der Barbarei, das es von allen Seiten umtobte (Temesvarer Zeitung 1882/190. 2).

Die Heterogenität der Bevölkerung, die Mehrzahl der deutsch sprechenden Stadtbewohner waren typische Charakteristika des Königreichs Ungarn, des „Mikrokosmos der großen zentraleuropäischen Region“ (Csáky 2010. 285). Auch im Falle von Temeswar herrschte, wie in anderen Städten des Königreichs Ungarn eine patriotische Haltung, die mehrmals gelobt wurde. Wie auch aus den Artikeln der untersuchten Zeitung wahrnehmbar ist, identifizierten sich die Nichtungarischsprachigen, vor allem die deutsch sprechende Bevölkerung mit der polyglotten Stadt, mit ihrem Komitat, mit dem Ort, an dem sie lebten 
und bewährten sich als ungarländische Patrioten, obwohl sie sich in mehreren kulturellen Kommunikationsräumen bewegten (Csáky 2010. 285). Ähnlich wie in den anderen urbanen Milieus (Preßburg, Czernowitz, Triest) der heterogenen zentraleuropäischen Region trafen auch in Temeswar ,unterschiedliche verbale und nonverbale Kommunikationsräume aufeinander, bildeten Enklaven, verschränkten sich aber auch, gingen ineinander über und trugen zu einer dynamischen, performativen, hybriden neuen städtischen Kultur bei“ (Csáky 2010. 307). Als Folge solcher Veränderungen im urbanen Milieu und steter Kontakte der anwesenden Nationen blühte Temeswar schön auf, überflügelte viele andere Städte, was dazu führte, dass Temeswar mit vollem Rechte die zweite Stadt Ungarns genannt wurde:

Man braucht nur einen aufmerksamen Blick auf seine Bevölkerung zu werfen, um zu erkennen, daß man sich in einer Stadt von vollkommen modern-europäischem Charakter [Hervorhebung von mir - J. E.] befindet, wo kein Anspruch auf irgend ein geistiges Bedürfniß, auf irgend eine Errungenschaft der Kultur unbefriedigt bleibt (Temesvarer Zeitung 1882/213. 1).

Interessanterweise erschien in diesen Jahren im ungarischen Tagesblatt Délmagyarországi Lapok dasselbe Großstadtbild über Temeswar, wenn auch nur im Traum. Im Feuilletonteil wurde eine Plauderei mit dem Titel Száz év múlva [In hundert Jahren] und Untertitel Olyan „Temesvári séta“-féle [Eine Art Temesvarer Spaziergänge $]^{5}$ veröffentlicht, in dem der Mitarbeiter der Zeitung Miklós Lőwy über seinen Traum berichtet, als er einem Gast aus Budapest die „,bekannteste und berühmteste Stadt Südungarns“ Temeswar zeigte. Auf den Straßen gab es prächtiges Pflaster, die Wagen verkehren ohne Lärm und die Prachtbauten bewunderten den hauptstädtischen Gast. Lőwy konnte dem Fremden eine kulturell, wirtschaftlich und wissenschaftlich auf hohem Niveau stehende Stadt vorstellen, wo die Bibliotheken zu jeder Zeit voll sind, wo die Südungarische Wissenschaftliche Akademie seit Langem existiert, wo ein ständiges ungarisches Theater ist und wo mehrere ungarische Tagesblätter und wissenschaftliche Zeitschriften lebten. $\mathrm{Ob}$ diese Wunschliste in hundert oder tausend Jahren verwirklicht wird, bleibt eine offene Frage. ${ }^{6}$

5 Száz év múlva [In hundert Jahren]. Olyan „Temesvári séta“-féle“ [Eine Art Temesvarer Spaziergänge]. In: Délmagyarországi Lapok Nr. 6 v. 9. Jänner 1881. 1.

6 „Most attól tartok, hogy ilyen sajátságos álomról szóló czikk fölé a »Száz év múlva « czím helyett nem jobban illenék-e ez a czím: »Ezer év múlva«.” Délmagyarországi Lapok Nr. 6 v. 9. Jänner 1881. 1. 


\section{Literaturverzeichnis}

Csáky, Moritz 2010. Das Gedächtnis der Städte. Kulturelle Verflechtungen - Wien und die urbanen Milieus in Zentraleuropa. Wien-Köln-Weimar, Böhlau.

Eckstein, Ernst 1876. Beiträge zur Geschichte des Feuilletons. 2. Aufl. Leipzig. Zit. nach: Jäger, Georg 1988: Das Zeitungsfeuilleton als literaturwissenschaftliche Quelle. Probleme und Perspektiven seiner Erschließung. In: Jäger, Georg - Martens, Wolfgang: Bibliographische Probleme im Zeichen eines erweiterten Literaturbegriffs. Weinheim, VCH Acta Humaniora, 53-73.

Ehrenpreis, Petronilla 2006. Die „reichsweite” Zeitungen in der Habsburgermonarchie. In: Rumpler, Helmut - Peter, Urbanitsch (Hg.): Die Habsburgermonarchie 1848-1918. Bd. 8: Politische Öffentlichkeit und Zivilgesellschaft. 2. Teilband, Wien, 1715-1818.

Fried, István 2001. Die Kleinstadt der Monarchie als Kulturelles und/oder Literarisches Gedächtnis in Ostmitteleuropa. Neohelicon 28 (1). 123-128.

Kauffmann, Kai 2000. „Narren der modernen Kultur”. Zur Entwicklung der Wochenplauderei im Wiener Feuilleton 1848-1890. In: Arman, K.-Lengauer, H.Wagner, K. (Hg.): Literarisches Leben in Österreich. Wien, Böhlau, 343-359.

Stöber, Rudolf 2005. Deutsche Pressegeschichte. 2. Aufl. Konstanz, UVK.

Todorow, Almut 1996. Das Feuilleton der „Frankfurter Zeitung” in der Weimarer Republik. Zur Grundlegung einer rhetorischen Medienforschung. Tübingen, Max Niemayer.

Tóth, Benedek 2003. Élet és/vagy irodalom. A heti csevegés (tárca) a 19. század második felének elkülönülő sajtórendszerében. [Leben und/oder Literatur. Die Wochenplauderei (Feuilleton) im sich differenzierenden Pressewesen der zweiten Hälfte des 19. Jahrhunderts]. In: Dajkó, Pál - Labádi, Gergely (Hg.): Klasszikus magyar irodalomtörténet. Szeged, Tiszatáj Könyvek, 215-230.

Tóth, Benedek 2014. A Wochenplauderei mint beszédmód (Egy lehetséges megközelítés). [Die Wochenplauderei als Diskurs. Eine mögliche Annäherung]. Magyar Könyvszemle 130. 452-471.

\section{Artikel aus der Temesvarer Zeitung}

Eine Vorstellung in optima forma. Temesvarer Zeitung Nr. 115 vom 20. Mai 1871. Temesvarer Genrebilder. Temesvarer Zeitung Nr. 116 vom 21. Mai 1871. Temesvarer Genrebilder. Temesvarer Zeitung Nr. 119 vom 25. Mai 1871. Temesvarer Plaudereien. Temesvarer Zeitung Nr. 122 vom 28. Mai 1871. Temesvarer Genrebilder (Musikalische Leiden). Temesvarer Zeitung Nr. 147 vom 29. Juni 1871.

Temesvarer Genrebilder. Temesvarer Zeitung Nr. 167 vom 23. Juli 1871. Federzeichnungen aus der Provinz. Temesvarer Zeitung Nr. 185 vom 13. Aug. 1871. 
Hahn, Ignaz: Temesvarer Schattenbilder. Temesvarer Zeitung Nr. 15 vom 20. Jänner 1872.

Das Parkfest. Temesvarer Zeitung Nr. 148 vom 2. Juli 1872.

Temesvarer Plaudereien. Temesvarer Zeitung Nr. 199 vom 1. Sept. 1872.

Briefe eines Müssiggängers. Temesvar als Großstadt. Temesvarer Zeitung Nr. 247 vom 27. Okt. 1872.

Temesvarer Plaudereien. Temesvarer Zeitung Nr. 154 vom 6. Juli 1873.

Temesvarer Plaudereien. Temesvarer Zeitung Nr. 180 vom 8. Aug. 1875.

Temesvarer Plaudereien. Temesvarer Zeitung Nr. 273 vom 28. Nov. 1875.

Spazierfahrten. In: Temesvarer Zeitung Nr. 252 vom 4. Nov. 1877.

Temesvarer Schattenbilder. Temesvarer Zeitung Nr. 23 vom 27. Jänner 1878.

A Bánság az anyaországhoz való visszacsatolásának százados ünnepére - Die Centennariumfeier der Reinkorporirung des Banates. Temesvarer Zeitung Nr. 93 v. 23. Apr. 1879.

Temesvarer Bilder und Geschichten. Temesvarer Zeitung Nr. 179 v. 6. August 1882.

Ein Jubiläum der Stadt Temesvar. Temesvarer Zeitung Nr. 190 v. 20. August 1882. Zu den Temesvarer Festtagen. Temesvarer Zeitung Nr. 213 v. 17. Sept. 1882.

Sternberg, Adolf: An das Temesvarer Lesepublikum. In: Temesvarer Zeitung Nr. 186 vom 15. August 1886. 